\title{
EDITORIAL
}

\section{SEMINARIO SOBRE LA ELIMINACIÓN \\ DEL SARAMPIÓN Y LA RUBEOLA EN ESPAÑA}

\author{
José Javier Castrodeza Sanz
}

Director General de Salud Pública, Calidad e Innovación. Ministerio de Sanidad, Servicios Sociales e Igualdad.

El sarampión es una enfermedad infecciosa altamente transmisible que, a pesar de poder prevenirse mediante vacunación, es una de las causas importantes de morbilidad y mortalidad infantil en el mundo. La rubéola es una enfermedad generalmente leve cuando aparece en la infancia pero que puede causar el síndrome de rubéola congénita en el recién nacido, cuando la infección ocurre durante el periodo fetal por infección en las mujeres en las primeras fases del embarazo. Esta enfermedad es muy grave y ocasiona secuelas e incluso la muerte.

La organización Mundial de la Salud (OMS) ha establecido que tanto el sarampión como la rubéola cumplen con los requisitos para que ambas enfermedades puedan ser eliminadas y por tanto ha apostado por la coordinación para su consecución.

En el año 2010, los ministros de sanidad de los países de la Región Europea de la Organización Mundial de la Salud renovaron su compromiso de eliminar el sarampión y la rubeola en la reunión del Comité Regional Europeo de la OMS, estableciendo la meta para el año 2015. Aunque no parece factible cumplir la meta establecida, la Oficina Europea de la OMS está realizando diversas actividades para sensibilizar a los estados miembros, entre las que se encuentran la organización de reuniones técnicas para tratar aspectos puntuales así como la puesta en marcha del proceso de verificación con notificaciones y recomendaciones sobre aspectos puntuales a cada país.

La Oficina Europea de la OMS solicitó realizar una visita técnica a España para conocer de primera mano el trabajo que se realiza en torno a la eliminación del sarampión y rubeola. La visita tuvo lugar los días 16, 17 y 18 de junio de 2015 y, como parte de la misma, se organizó un seminario el día 17 en el salón de actos del Ministerio de Sanidad, Servicios Sociales e Igualdad. En él participaron los representantes de las comunidades autónomas encargados de los programas de vacunación, de la vigilancia epidemiológica y de actividades de laboratorio. También se solicitó la participación de las asociaciones de profesionales cuya actividad tiene relación con la detección de la enfermedad y su notificación y el establecimiento de medidas para la prevención y control del sarampión y la rubeola.

Durante el desarrollo del seminario se presentó una actualización de la situación en el mundo y en la Región Europea de la OMS 
con respecto a estas enfermedades y las estrategias y actividades recomendadas para su eliminación. Además se presentó el trabajo que se desarrolla desde el Ministerio de Sanidad, el Instituto de Salud Carlos III y las comunidades autónomas. La reunión finalizó con una interesante discusión entre todos los presentes.

Este monográfico de la Revista Española de Salud Pública recoge las ponencias presentadas durante el seminario. En los artículos se refleja la situación y el progreso realizado en la Región Europea de la OMS y las principales actividades que se desarrollan en España para conseguir las metas fijadas. Se recoge la situación actual en España y las recomendaciones para continuar avanzando hacia la eliminación de ambas enfermedades.

Quiero agradecer a todas las personas e instituciones que colaboraron en la organización y desarrollo de las reuniones mantenidas con la delegación de la Oficina Europea de la OMS y, de manera especial, a las personas que ayudaron en la organización de las reuniones, es decir, a la Subdirectora de Promoción de la Salud y Epidemiología, Elena Andradas Aragonés, y a las personas del Área de Programas de Vacunación, Aurora Limia Sánchez, Marta Molina Olivas, Soledad Cañellas Llabrés y Dolores Rodrigo Muñoz.

Muchas gracias. 